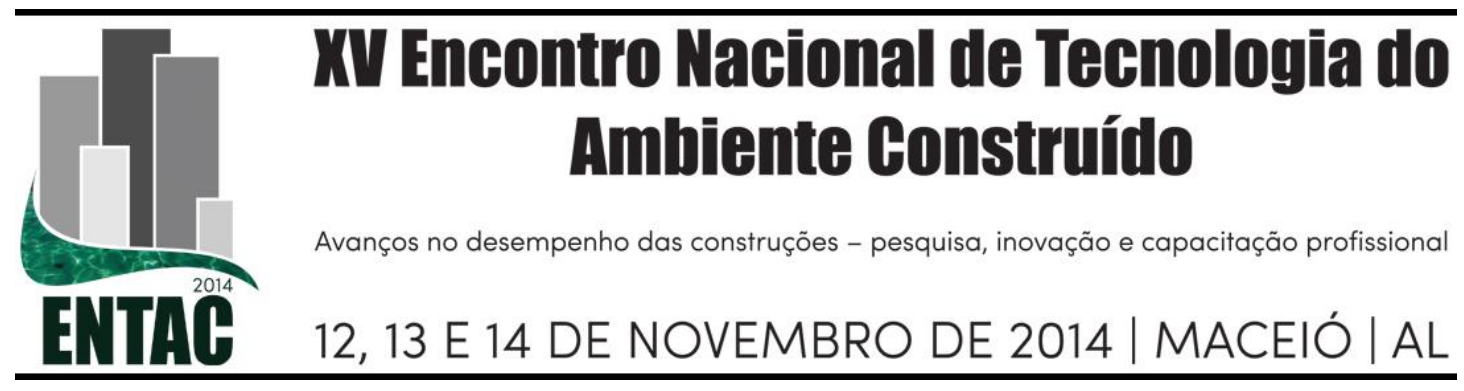

\title{
CORRELACIÓN ENTRE LAS SENSACIONES TÉRMICAS DE AULAS Y LA RESPUESTA CONDUCTUAL DE LOS NIÑOS DE 5 AÑNOS
}

\section{Ms. GARCÍA CARDONA, Ader (1); Dc. MATAMOROS TUMA, Mabel (2) Dc. ALFONSO GONZÁLEZ, Alfonso (3)}

(1) Universidad Nacional de Colombia, e-mail: agarcia@unal.edu.co, (2) Instituto Técnico Superior José Antonio Echeverría, e-mail: mabel@arquitectura.cujae.edu.cu, (3) Universidad San Gerónimo, La

Habana, Cuba, e-mail: alfonso@sangeronimo.ohc.cu

\begin{abstract}
RESUMEN
Medellín, Colombia, es una ciudad andina con un promedio de temperatura entre los 16 y $\operatorname{los} 29^{\circ} \mathrm{C}$, con una variación de la humedad relativa entre 30 y $70 \%$ condiciones que hacen difícil conseguir confort térmico en las aulas de los jardines infantiles. Esta investigación busca determinar la posible influencia, en actividades que realizan los niños, de las condiciones térmicas del aula. Se midió la sensación térmica en seis aulas de jardines infantiles de la ciudad de Medellín, tres de esas aulas fueron construidas siguiendo la norma técnica vigente en Colombia, NTC 4595, (MEN, COLOMBIA, 2006) y las otras tres antes de la misma. Las medidas se realizaron en dos días diferentes del año: uno en el mes más frío y otro en el mes más cálido mientras los niños dibujaban. Paralelo a las medidas térmicas del aula se puso a los niños a realizar actividades que obligaran su atención y concentración, procesos psicológicos básicos (PSB), y se correlacionaron los resultados mediante los coeficientes de correlación de Pearson. Esas correlaciones indicaron que el calentamiento rápido y la inercia térmica afectan negativamente el desempeño en los procesos psicológicos básicos de los niños al interior del aula. El uso de las correlaciones permite identificar relaciones entre datos obtenidos de la metodología de observación cualitativa y los datos termodinámicos. se usaron para observar los procesos psicológicos básicos puede existir alguna relación inversa entre la atención y la concentración de los niños con la velocidad de calentamiento del aula, que a su vez tiene que ver con la inercia térmica de las paredes del aula y las renovaciones de aire.
\end{abstract}

Palabras-clave: Sensación térmica, Conducta de los niños, Aulas Jardines infantiles.

\begin{abstract}
Medellin is a dean tropical city with an average Temperature between $16^{\circ} \mathrm{C}$ to $29^{\circ} \mathrm{C}$, and a Relative Humidity between $30 \%$ to $70 \%$, conditions which make it difficult to achieve comfort for the children in kindergarte classrooms. This investigation aims to determine the possible influence of the classroom's thermal performance, in the behavior of Children. Six classrooms were measured, whit both warm and cold weather. Three of those classrooms were build before the NTC 4595, (MEN, COLOMBIA, 2006) , Technical Standard for the construction of school environments. The other three, were build under the parameters described in the mention standard.The measures has been doing in two diferent wheather days: hot and cold day while the children make a skecht. In the same time make application of standard cognitive test, the basic psychological processes (BPP), and calculate the Pearson's correlation coefficient statistical test. In fact, it was observed that the faster heat and thermical inertia are gain in the interior of the classroom wall and air renovation, the greater the loss, in the BPP efficiency. To calculate the correlation, a qualitative observational methodology was used for the evaluation of the performance of BPP, and to obtain the thermodynamic data, quantitative measurements were taken. Both types of data were crossed by the Pearson's correlation coefficient statistical test.
\end{abstract}




\section{INTRODUCCIÓN}

Colombia es un país localizado en el trópico sobre el eje norte-sur atravesado por tres cadenas montañosas de los Andes, situación geográfica que produce una variabilidad climática compleja, confirmando que el control térmico de los edificios es obligatorio para lograr la comodidad de los usuarios. Esa obligación aumenta cuando se trata de un edificio destinado a un jardín infantil por las características físicas y psicológicas de los niños y los métodos pedagógicos aplicados.

Para 2010, Medellín, Colombia contaba con una población total de 2’343.049 habitantes, de la cual 205.652 eran menores de 6 años, un $71 \%$ de ellos, menores de 5 años, vivían en condiciones de vulnerabilidad (HERRERA R, 2012). Los edificios hechos para atender a la primera infancia se construyen con normas que no hacen precisiones técnicas sobre el comportamiento térmico de las aulas por causa de las condiciones pedagógicas y fisiológicas de los niños, distintas a la de los otros niveles educativos. En consecuencia, es necesario identificar si las condiciones termodinámicas de las aulas tienen alguna incidencia en el desempeño de los niños.

\section{DESARROLLO}

\subsection{Marco teórico}

Las investigaciones específicas sobre condiciones del ambiente térmico de las aulas se encargan de medir la respuesta de los niños tratando de aplicar metodologías validadas para adultos: El trabajo realizado por Fabbri, (2013) interesado en conocer la respuesta de los niños de 4 y 5 años de un Jardín infantil al comportamiento Térmico del Aula, adaptando las metodologías del PMV y PPD al nivel cognitivo de los niños, advierte en los resultados que los niños de esa edad son capaces de emitir conceptos claros sobre su entorno térmico y que las zonas de confort se deben revisar para cada grupo de edad. Teli; Jentsch; James, (2012) identifican que los niños de 7 a 11 años son mucho más sensibles a las altas temperaturas que los adultos, razón por la cual el control de rangos es distinto de acuerdo a la edad de las personas. Mors et al., (2011) y Humphreys, (1977) trabajan sobre el confort adaptativo de los niños en las aulas con el cambio de estaciones y los índices de arropamiento (clo).Wargocki; Wyon, (2007) midieron los cambios en las respuestas de alumnos de 10 a 12 años a ejercicios de lecto-escritura y matemáticas según como modificaban la temperatura del aire del aula con un apoyo de aire acondicionado. Estas respuestas mejoraban según se hacía más baja la temperatura si era verano, pero advierte de que en algunos países sin estaciones el incremento en la temperatura podría mejorar el desempeño académico de los niños debido a la aclimatación. García, Martinez (2003) advierten que los niños de cinco años presentan dificultades para distinguir entre las condiciones térmicas y las lumínicas del aula y que sus opiniones sobre las ambientales se acercan a más a criterios estéticos holísticos

Conforme a lo anterior todas las investigaciones consultadas se realizan en lugares con estaciones en los que es normal que periodos cálidos húmedos coincidan con las vacaciones escolares, siendo inapropiado asimilar esas observaciones a las aulas en países tropicales. En el caso de países sin estaciones hay muchos lugares con clima cálido húmedo durante todo el año y la adaptabilidad del niño a esas condiciones es mayor. 
Por otro lado la normatividad técnica colombiana para diseño y construcción de edificaciones educativas, NTC4595, (MEN, COLOMBIA, 2006) indica en la tabla 8 del numeral 7.3.5. las condiciones de la ventilación para las aulas según tres tipos de clima, pero ninguna se ajusta a las condiciones de Medellín, Colombia, que presenta una variación térmica promedio entre $\operatorname{los} 16^{\circ} \mathrm{C}$ y los $29^{\circ} \mathrm{C}$ y una fluctuación en la humedad relativa entre 30 y $80 \%$, temperaturas altas para clima templado y humedades bajas para el clima cálido húmedo. Con esta situación se expone a los niños a mayor variabilidad adaptativa durante su jornada académica en el aula.

En consecuencia, es necesario identificar si las condiciones termodinámicas de las aulas tienen alguna incidencia en el desempeño de los niños.

\subsection{Objetivo}

Encontrar relaciones entre las conductas de los niños en las aulas de los jardines infantiles y las sensaciones térmicas medidas a partir de los registros de temperatura de bulbo seco, temperatura de globo, humedad relativa y velocidad de viento.

\section{MÉTODO}

\subsection{Medidas Higrotérmicas}

Se realiza un medición en seis aulas diseñadas para jardines infantiles en Medellín, Colombia, ver Figura 1, tres de ellas construidas antes de la norma NTC 4595 (MEN, COLOMBIA, 2006) y tres construidas después de la misma. Se tomaron datos de temperatura de bulbo seco, humedad relativa y velocidad del aire en el mismo momento en que se realizaba una observación de la respuesta conductual de niños de cinco años.

Las características de selección de las aulas fueron: orientación de las ventanas para iluminar y ventilar en fachadas sur y norte, altitud sobre el nivel de mar entre los 1450 y 1950 metros, relación entre área de vanos y área de piso: $1 / 6$, área del aula 35 y $40 \mathrm{~m}^{2}$, una altura libre de $2.5 \mathrm{~m}$, capacidad de 20 a 25 niños y horario de escolar de $8 \mathrm{am}$ a $4 \mathrm{pm}$. 
Figura 1 - Identificación de las aulas
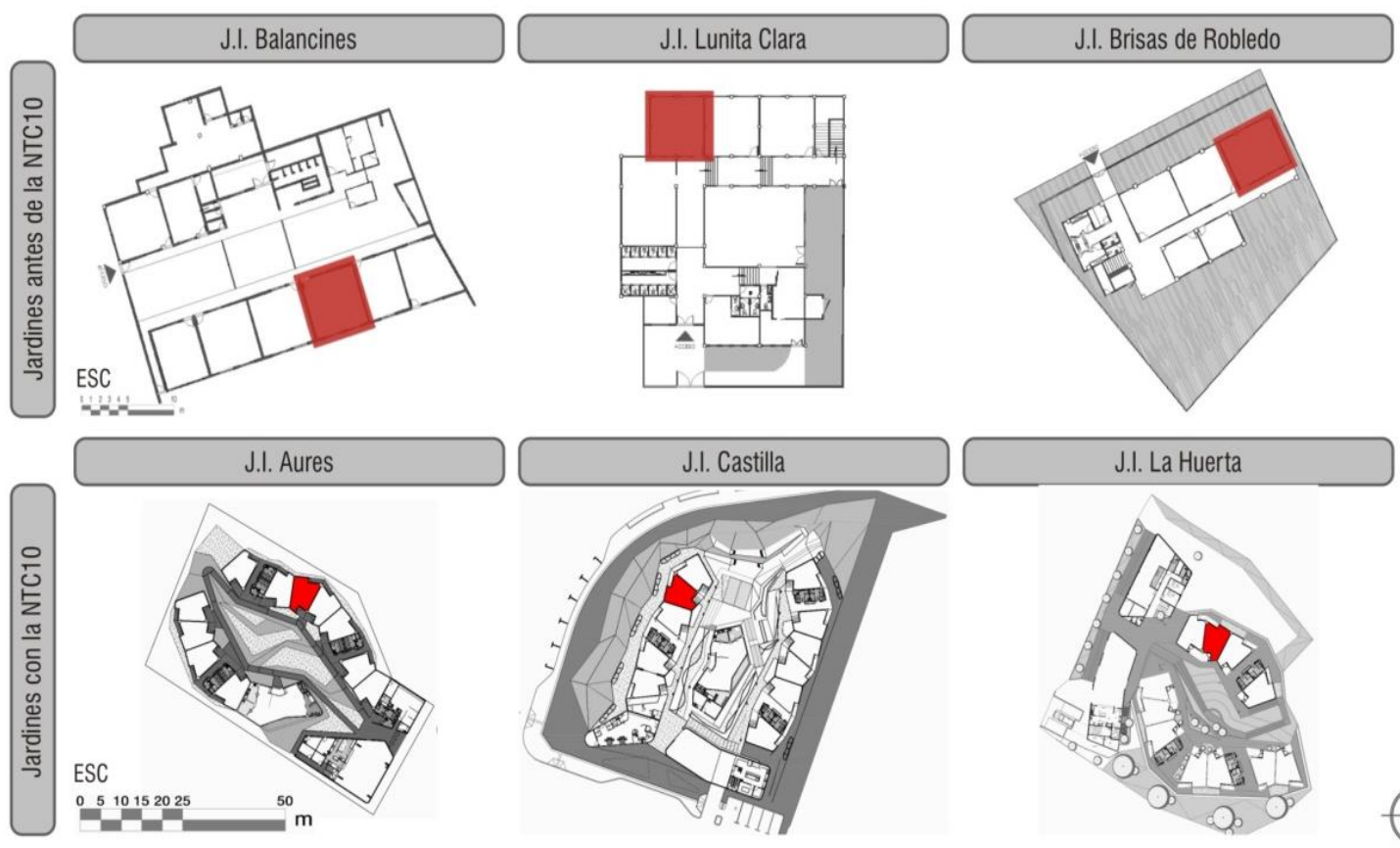

Se siguen los procedimientos técnicos descritos en las normas ISO 7243:1989, $2006^{1}$ y se miden las sensaciones térmicas a partir de los datos de Temperatura de Bulbo Seco (TBS), Humedad Relativa (\%), Temperatura de Globo (TG) y Velocidad del Viento $(\mathrm{m} / \mathrm{s})$. Los equipos de medida se programan para hacer mediciones durante una semana iniciando el día viernes a las 12:00m, con registro de datos cada 10 minutos. Las medidas se realizan en el mes con promedios más altos de temperatura: Agosto y el mes con promedios más bajos de temperatura: Octubre como se muestra en el Cuadro 1.

\section{Cuadro 1- Comportamiento climático de Medellín}

\begin{tabular}{|c|c|c|c|c|c|c|c|c|c|c|c|c|}
\hline \multicolumn{13}{|c|}{ Tabla Climatológica de Medellín, Colombia } \\
\hline \multicolumn{13}{|c|}{ Temperatura $\left({ }^{\circ} \mathbf{C}\right)$} \\
\hline Mes & Ene & Feb & Mar & Abr & May & Jun & Jul & Ago & Sep & Oct & Nov & Dic \\
\hline $\begin{array}{l}\text { Mínima } \\
\text { promedio }\end{array}$ & 16,1 & 16,3 & 16,7 & 17 & 17 & 16,6 & 16,1 & 16,3 & 16,2 & 16,3 & 16,4 & 16,3 \\
\hline Promedio & 22 & 22,3 & 22,3 & 22 & 21,9 & 22,3 & 22,5 & 22,5 & 21,9 & 21,2 & 21,2 & 21,5 \\
\hline $\begin{array}{l}\text { Máxima } \\
\text { promedio }\end{array}$ & 28,1 & 28,5 & 28,5 & 27,9 & 27,8 & 28,1 & 28,6 & 28,5 & 28 & 27,2 & 27,3 & 27,6 \\
\hline \multicolumn{13}{|c|}{ Precipitación, brillo solar y humedad relativa } \\
\hline Mes & Ene & Feb & Mar & Abr & May & Jun & Jul & Ago & Sep & 0ct & Nov & Dec \\
\hline \begin{tabular}{l|} 
Precipitación \\
promedio $(\mathrm{mm})$
\end{tabular} & 55 & 77 & 114 & 179 & 191 & 153 & 108 & 154 & 178 & 218 & 150 & 79 \\
\hline Días Iluvia & 11 & 12 & 16 & 21 & 23 & 18 & 16 & 19 & 21 & 24 & 21 & 14 \\
\hline $\begin{array}{l}\text { Humedad } \\
\text { relativa (\%) }\end{array}$ & 66 & 66 & 67 & 70 & 71 & 68 & 63 & 64 & 68 & 72 & 73 & 70 \\
\hline $\begin{array}{l}\begin{array}{l}\text { Brillo Solar } \\
\text { (horas/mes) }\end{array} \\
\end{array}$ & 177 & 148 & 154 & 128 & 142 & 170 & 204 & 192 & 150 & 135 & 140 & 156 \\
\hline \multirow{4}{*}{\multicolumn{3}{|c|}{$\begin{array}{c}\text { Datos Mededidos en: } \\
\text { Aeropuerto Olaya Herrera } \\
\text { IDEAM }\end{array}$}} & \multirow{4}{*}{\multicolumn{2}{|c|}{$\begin{array}{c}\text { Promedios } \\
\text { Anuales }\end{array}$}} & \multicolumn{3}{|c|}{ Temperatura } & \multicolumn{4}{|c|}{ Precipitación } & Brillo \\
\hline & & & & & Min & Med & $\operatorname{Max}$ & Total & Lluvia & \multicolumn{2}{|c|}{ Humedad } & Solar \\
\hline & & & & & ${ }^{\circ} \mathbf{C}$ & ${ }^{\circ} \mathrm{C}$ & ${ }^{\circ} \mathbf{C}$ & $\mathrm{mm}$ & Días & \multicolumn{2}{|c|}{$\%$} & horas \\
\hline & & & & & 16,4 & 22 & 28 & 1656 & 215 & \multicolumn{2}{|c|}{68} & 158 \\
\hline
\end{tabular}

\footnotetext{
${ }^{1}$ ISO 7243:1989. Hot environments. Estimation of the heat stress on working man, based on the WBGT index (wet bulb globe temperature). 2006 p. 1-20.
} 


\subsection{Medidas de la respuesta conductual de los niños}

La observación de la conducta en los niños es una práctica común en la investigación clínica y del aprendizaje en psicopedagogía. Permite encontrar relación entre aspectos de la conducta, llamados procesos psicológicos básicos (PPB) como: la atención, concentración y motivación, de los niños y condiciones del ambiente donde desarrollan sus actividades. Los procedimientos observacionales de los PPB se construyen desde objetos de análisis que varían en su complejidad a partir de las características como: la frecuencia de la ocurrencia de la conducta, la intensidad de la conducta y la duración, entre otras características. (SATTLER, 2008)

Para la determinación de la observación de los PPB se seleccionan las CONDUCTAS META, que deben ajustarse a los objetivos de la investigación: 1) Seguir la línea: Se define como la acción de seguir con el lápiz de color las líneas de contorno de los dibujos presentados y 2) Uniformidad del trazo: Se definió como la acción de rellenar las zonas del dibujo con trazos del mismo tamaño, intensidad y dirección hasta completar la zona dibujada.

Se definió que los niños realicen la actividad de "Pintar" dos veces en un día, dos días en el año: la primera, en un día caluroso (17 de Agosto) y la segunda para un día frío (19 de Octubre). Los intervalos horarios previstos para las actividades son: en la mañana de 9 a 10am y en la tarde de 1:30 a 2:30pm. La actividad debe duró15 minutos dentro de los cuales se hizo registro de eventos durante 5 minutos, una vez cada minuto se registró el número de ocurrencia de las Conductas Meta descritas.

Las aulas no tuvieron ninguna modificación física, las pruebas se realizaron con las ventanas abiertas y las luces de las aulas apagadas. Los niños se acomodaron dentro del aula en el lugar que prefirieron. 


\section{RESULTADOS}

\section{Medidas de la respuesta conductual de los niño Correlaciones entre las sensaciones térmicas y la respuesta conductual de los niños}

En el Cuadro 2 se presentan con los resultados de las conductas metas registradas: Seguir la línea (SL) y Uniformidad (U) analizadas mediante el coeficiente de correlación de Pearson con las sensaciones térmicas (ST) medidas en los momentos de las pruebas donde se identifican en color verde las correlaciones negativas y en naranja las correlaciones positivas.

\section{Cuadro 2- Correlaciones entre conductas meta y sensaciones térmicas}

\begin{tabular}{|c|c|c|c|c|c|c|c|c|}
\hline & & Aula & Total & SL & $\mathbf{U}$ & ST & $\begin{array}{c}\text { Correl SL- } \\
\text { ST }\end{array}$ & $\begin{array}{c}\text { Correl U- } \\
\text { ST }\end{array}$ \\
\hline \multirow{6}{*}{$\begin{array}{l}\sum \\
\Sigma \\
0 \\
\text { s } \\
0 \\
0 \\
\dot{c}\end{array}$} & is & Balancines & 27 & 2 & 2 & 23 & \multirow{3}{*}{0,37} & \multirow{3}{*}{0,34} \\
\hline & 음 & Brisas de Robledo & 20 & 6 & 7 & 23 & & \\
\hline & $>$ & Lunita Clara & 20 & 1 & 1 & 22 & & \\
\hline & $\approx$ & Aures & 22 & 8 & 7 & 21 & \multirow{3}{*}{$-0,61$} & \multirow{3}{*}{$-0,66$} \\
\hline & d & La Huerta & 20 & 5 & 9 & 21 & & \\
\hline & $z^{3}$ & 12 de 0 ctubre & 27 & 5 & 6 & 24 & & \\
\hline \multirow{6}{*}{$\begin{array}{l}\sum \\
a \\
0 \\
0 \\
\dot{s} \\
0 \\
0 \\
\alpha\end{array}$} & \multirow{3}{*}{ 옹 } & Balancines & 27 & 2 & 2 & 27 & \multirow{3}{*}{0,34} & \multirow{3}{*}{$-0,64$} \\
\hline & & Brisas de Robledo & 20 & 4 & 7 & 21 & & \\
\hline & & Lunita Clara & 20 & 0 & 1 & 23 & & \\
\hline & s & Aures & 15 & 8 & 2 & 26 & \multirow{3}{*}{$-0,66$} & \multirow{3}{*}{$-0,5$} \\
\hline & ఖ & La Huerta & 15 & 4 & 4 & 24 & & \\
\hline & $\sum^{3}$ & 12 de 0 ctubre & 25 & 2 & 3 & 24 & & \\
\hline \multirow{6}{*}{ 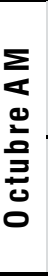 } & $\infty$ & Balancines & 27 & 2 & 2 & 21 & \multirow{3}{*}{0,87} & \multirow{3}{*}{0,98} \\
\hline & \multirow{2}{*}{$\stackrel{\stackrel{0}{\circ}}{\stackrel{0}{\infty}}$} & Brisas de Robledo & 21 & 9 & 6 & 20 & & \\
\hline & & Lunita Clara & 22 & 2 & 0 & 20 & & \\
\hline & s & Aures & 18 & 3 & 2 & 19 & \multirow{3}{*}{0,24} & \multirow{3}{*}{$-0,39$} \\
\hline & d & La Huerta & 24 & 11 & 16 & 20 & & \\
\hline & $\sum^{3}$ & 12 de 0 ctubre & 16 & 8 & 0 & 21 & & \\
\hline \multirow{6}{*}{$\begin{array}{l}\sum_{0} \\
0 \\
0 \\
0 \\
0 \\
\vdots \\
0 \\
0\end{array}$} & \multirow{6}{*}{$\frac{\stackrel{\infty}{.0}}{\stackrel{.0}{>}}$} & Balancines & 26 & 11 & 7 & 24 & \multirow{3}{*}{0,91} & \multirow{3}{*}{0,95} \\
\hline & & Brisas de Robledo & 20 & 9 & 5 & 22 & & \\
\hline & & Lunita Clara & 22 & 0 & 0 & 20 & & \\
\hline & & Aures & 10 & 0 & 0 & 20 & \multirow{3}{*}{0,27} & \multirow{3}{*}{0,13} \\
\hline & & La Huerta & 24 & 16 & 20 & 19 & & \\
\hline & & 12 de 0 ctubre & 12 & 14 & 10 & 22 & & \\
\hline
\end{tabular}

En el mes de Agosto las correlaciones de las aulas nuevas son negativas durante las dos medidas del día, quiere decir que a medida que aumenta la sensación térmica disminuye el desempeño cognitivo de los niños, ese mismo comportamiento solo ocurre en la tarde de las aulas de los jardines viejos. 
Para el mes de Octubre el comportamiento es contrario: las aulas de los jardines viejos presentan correlaciones altas y positivas en las dos medidas del día, lo que indica que sí la sensación térmica aumenta también lo hará el desempeño de los niños.

Las correlaciones muestran un comportamiento opuesto entre las aulas de los jardines viejos y nuevos según el mes del año: en Agosto, el mes más caliente, las humedades relativas y las temperaturas de todas las aulas son casi idénticas, sin embargo, el incremento de la temperatura es más rápido en las aulas nuevas, pues su desfase térmico es menor por tener menos masa térmica, esto hace que la sensación térmica aumente más rápido que en las aulas de los jardines viejos, que se calientan lentamente y pierdan menos humedad. (Ver Figura 2 y 3 ).

Figura 2 - Comparado Temperaturas Agosto

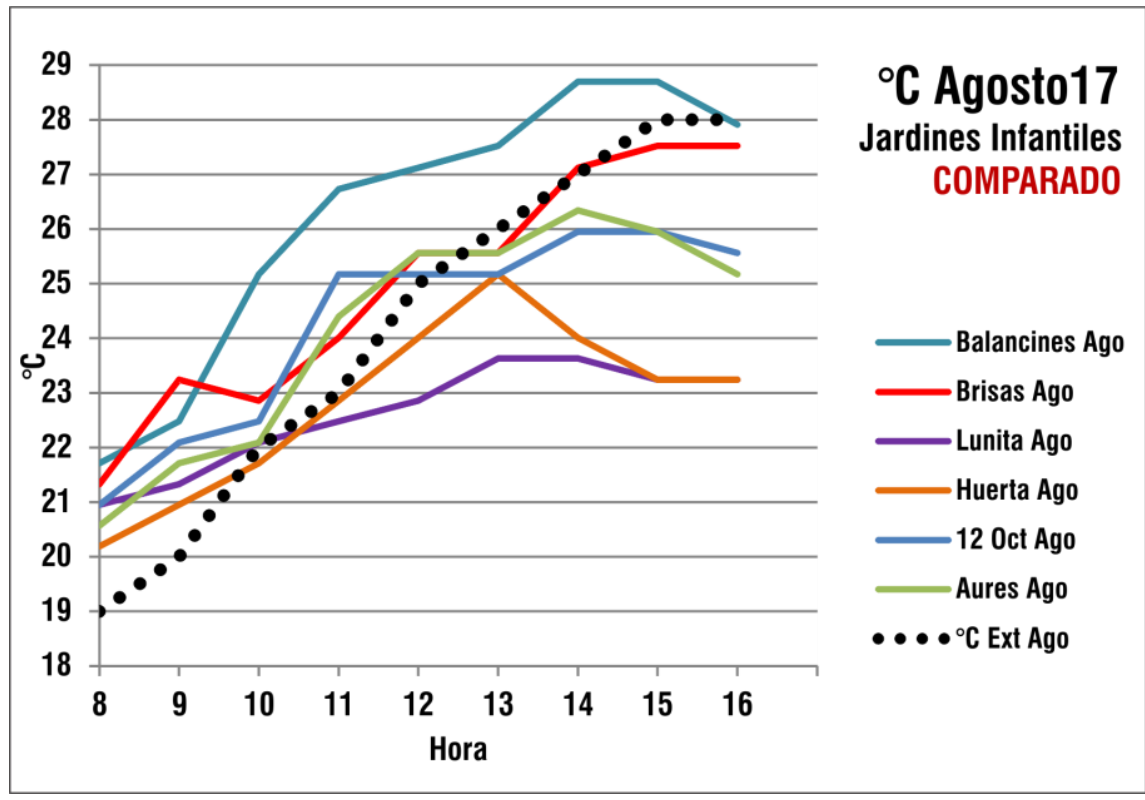

Figura 3 - Comparado Humedades Relativas Agosto

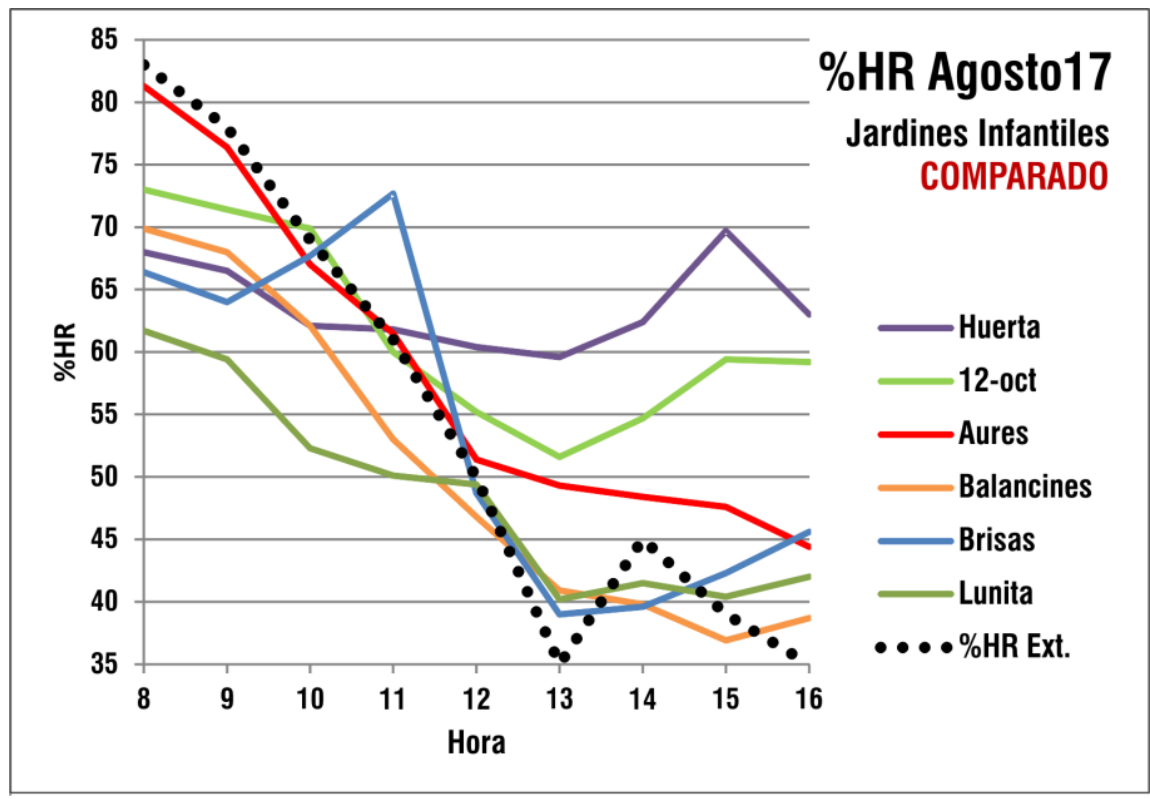


En octubre el comportamiento de las aulas es menos estable: mientras las aulas viejas mantienen casi la misma humedad relativa todo el día y un incremento de apenas un $1.2^{\circ} \mathrm{C}$ entre la mañana y la tarde, las aulas nuevas aumentan casi $1.5^{\circ} \mathrm{C}$ en el mismo periodo de tiempo, pero disminuyen $14.2 \%$ en la humedad relativa. Esto significa que la velocidad del viento es el elemento diferenciador en ambos casos. Las aulas viejas se ventilaron poco y como su velocidad de calentamiento es más lenta, la sensación térmica se mantuvo estable y baja durante todo el día, comportamiento consistente con la correlación presentada. (Ver Figura 4 y 5).

Figura 4 - Comparado Temperaturas Octubre

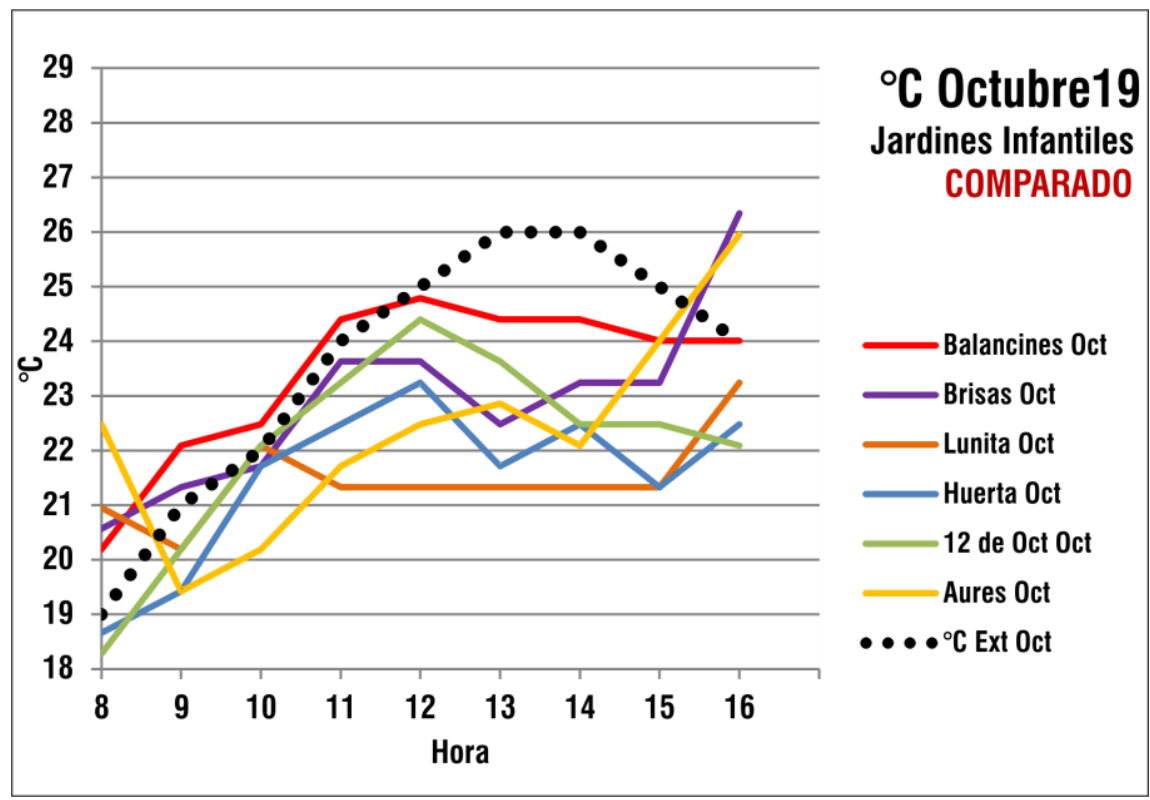

Figura 5 - Comparado Humedades Relativas Octubre

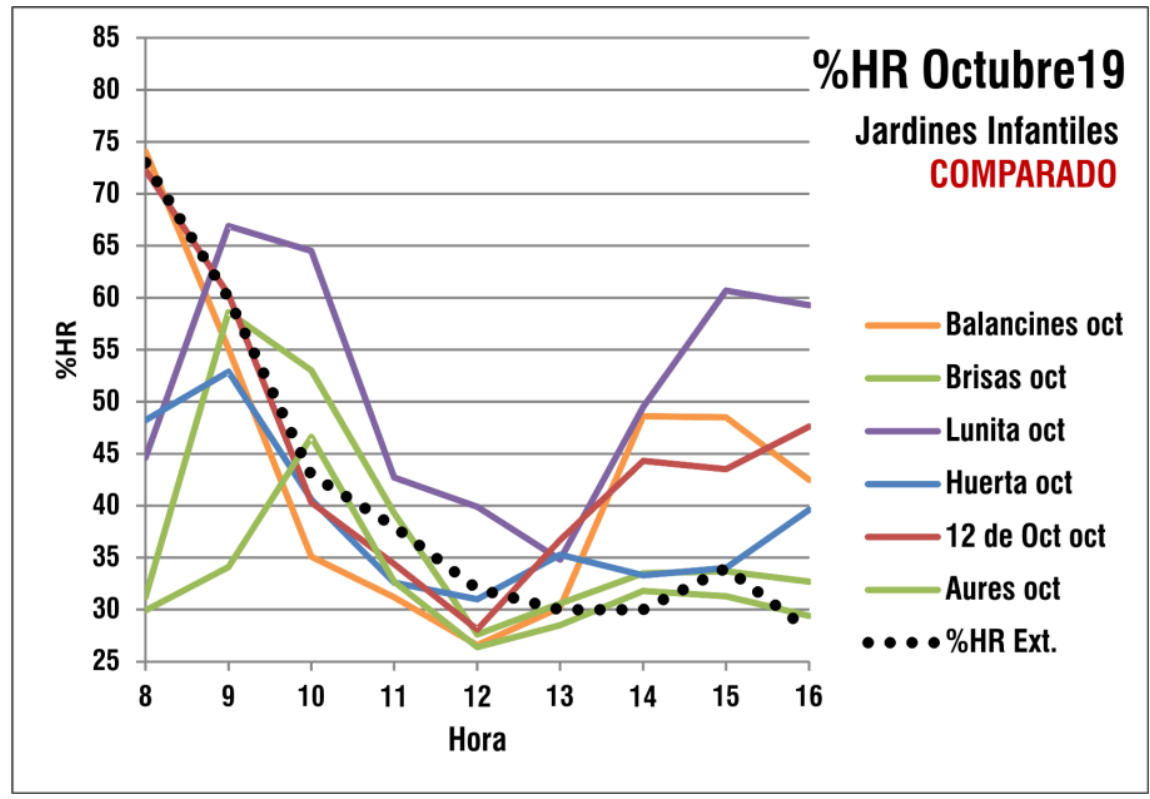


Se puede resumir diciendo que la sensación térmica funciona a favor o en contra del desempeño cognitivo de los niños en función de la velocidad de calentamiento del espacio y la ventilación, para condiciones cálidas con poca ventilación se debe disminuir la velocidad de calentamiento para hacer más lento el aumento de la sensación térmica y para condiciones frías aumentar la perdida de la humedad relativa con ventilación para aumentar la sensación térmica.

\section{CONCLUSIONES}

La estadística inferencial aplicada a este estudio prueba su fidelidad en la construcción de hipótesis investigativas que conectan observaciones cualitativas con cuantitativas, esquema que para estudios de arquitectura es valioso como herramienta de análisis y observación. No obstante, la interpretación de los resultados no siempre expresa de forma literal la relación entre los datos evaluados, en este caso, ninguna de las correlaciones se explica por la simple condición del aspecto evaluado.

Las conductas meta permitieron resolver el enfoque observacional y disminuir errores experimentales por la facilidad en identificarlas y la agilidad en los procesos de compilación y análisis.

Con la Sensación Térmica, las correlaciones son más complejas de explicar por qué obedecen a un fenómeno multivariado que involucra variables físicas medibles Temperatura de Bulbo Seco, Humedad Relativa, Velocidad del Viento, Inercia Térmica y variables subjetivas como la ropa, el clima y las actividades de los niños. No obstante, hay información general que pone en contexto los hallazgos encontrados.

El comportamiento Térmico entre las Aulas antes y después de la norma es diferenciado: las construidas antes de la norma son más calientes y húmedas pero con mayor Masa Térmica y las Aulas construidas después de la norma son más frescas y secas pero con poca masa térmica.

\section{REFERENCIAS}

HEERERA R, M. L. Documento de trabajo interno tema: políticas y programas de atención intengral a la primera infancia - la experiencia de medellín y el programa Buen Comienzo. Medellín, Colombia: Proantioquia. 2012.

FABBRI, K. Thermal Comfort Evaluation in Kindergarden: PMV and PPD measurement through datalogger and questionnaire. Building and Environment, v. Vol. 68, p. 202-214, 2013.

TELI, D.; JENTSCH, M. F.; JAMES, P. A. B. Naturally ventilated classrooms: An assessment of existing comfort models for predicting the thermal sensation and preference of primary school children. Energy and Buildings, v. Vol.53, p. 166-182, out. 2012. Disponível em: <http://linkinghub.elsevier.com>. Acesso em: 20 nov. 2013.

HUMPHREYS, M. A. A Study of Thermal Comfort of Primary School Children in Summer. Building and Environment, v. Vol. 12, p. 231-239, 1977.

ISO 7243:1989. Hot environments. Estimation of the heat stress on working man, based on the WBGT-index (wet bulb globe temperature). . [S.l: s.n.]. , 2006 
MEN COLOMBIA. Norma Técnica Colombiana NTC 4595 Ingeniería Civil y Arquitectura. Planeamiento y Diseño de Instalaciones y Ambientes Escolares. . [S.l: s.n.]. Disponível em: <www.mineducacion.gov.co>. , 2006

MORS, S. TER et al. Adaptive thermal comfort in primary school classrooms: Creating and validating PMV-based comfort charts. Building and Environment, v. Vol. 46, n. 12, p. 2454-2461, dez. 2011. Disponível em: <http://linkinghub.elsevier.com>. Acesso em: 10 nov. 2013.

SATTLER, J. M. Evaluación infantil. Aplicaciones conductuales, sociales y clínicas. [S.1.]: Manual Moderno, 2008. p. 1-678(Pág 216)

WARGOCKI, P.; WYON, D. P. The Effects of Moderately Raised Clasroom Temperature and Clasroom Ventilation Rate on the Performance of Schoolwork by Children. HVAC\&R Research, v. Vol. 13, n. 2, p. 193-220, 2007.

GARCÍA C., Ader A. y MARTÍNEZ, Fabio, 2003, Evaluación de la Actitud en los alumnos y Profesores de un Aula, antes y después de una Intervención Ergonómica en los Factores de Ruido, Iluminación y Temperatura. Universidad de Antioquia, Colombia. 2003 